\title{
Editorial
}

\section{Emerging Trends in Mobile Collaborative Systems}

\author{
Sergio F. Ochoa, ${ }^{1}$ Weiming Shen, ${ }^{2}$ Giancarlo Fortino, ${ }^{3}$ and Wenfeng $\mathrm{Li}^{4}$ \\ ${ }^{1}$ Department of Computer Science, University of Chile, 837-0456 Santiago, Chile \\ ${ }^{2}$ National Research Council of Canada, Ottawa, ON, Canada K1A OR6 \\ ${ }^{3}$ Department of Informatics, Modeling, Electronics and Systems, University of Calabria, 87036 Rende, Italy \\ ${ }^{4}$ School of Logistics Engineering, Wuhan University of Technology, Wuhan 430070, China
}

Correspondence should be addressed to Sergio F. Ochoa; sochoa@dcc.uchile.cl

Received 18 July 2016; Accepted 19 July 2016

Copyright (C) 2016 Sergio F. Ochoa et al. This is an open access article distributed under the Creative Commons Attribution License, which permits unrestricted use, distribution, and reproduction in any medium, provided the original work is properly cited.

During the last decades mobile collaborative systems have become transversal applications that provide services not only to end-users, but also to other systems like mobile social applications, context-aware recommender systems, monitoring systems, and crowd sourcing and participatory sensing applications. Providing support services in mobile collaboration scenarios is complex, because the work context of each participant could be different, and also such a context is dynamic. Moreover, these solutions involve a wide range of devices that should interoperate to provide particular services in heterogeneous scenarios, like in Internet of Things based working contexts.

Regardless the advantages that these systems bring to the mobile collaboration research and application domains, they also raise several challenges to system developers, particularly in terms of design, implementation, use, and evaluation of these solutions. This special issue covers some of the new trends and challenges of these systems, by presenting interesting articles that were accepted after a rigorous peerreviewing process.

One of the key issues in this computing scenario is the positioning of the nodes, since their mobility usually makes them change their work context and therefore the services that they can consume and provide to other nodes. Position accuracy (particularly in indoor environments), energy consumption, and deployment effort of these solutions are aspects highly researched by the community. In this sense, the paper by W. Kim et al. proposes a localization method named FreeLoc that deals with major technical issues in the crowdsourcing-based systems that involve indoor positioning. This method uses existing Wi-Fi infrastructure for performing the positioning and it does not require calibration (fingerprinting). This approach also considers the heterogeneity of devices that are usually available in a crowdsourcing scenario, which represents an important advance towards easy-to-deploy indoor positioning systems.

The paper by S.-H. Lee et al. also helps improve the positioning capability of these systems. It focuses on the positioning accuracy. The article proposes a beacon-based indoor positioning method, using an extended Kalman filter that recursively processes positioning information that also includes noise. This method improves the accuracy of indoor positioning techniques by fusing the information about the resources location with the data obtained from existing wireless indoor positioning technology. Thus, this method allows reaching average positioning errors that are between 26 and 28 centimeters.

The systems mentioned above usually have high power consumption since they exchange an important number of messages, not only to perform the positioning but also to support the mobile collaboration process. Many researches have already been done to reduce the energy consumption of mobile collaborative applications. In this sense, the paper by J. E. Luzuriaga et al. proposes an extension to the wellknown publish/subscribe scheme of the MQTT protocol, by introducing an intermediate buffer that takes care of message transfer. The experimental evaluation in a real environment shows that the nodes movement has a marginal impact in the message transfer. Particularly, this proposal guarantees that no information loss appears in the presence of variable length handoffs.

The paper by M. Chincoli et al. also explores the energy consumption from a different perspective. It analyzes the conditions under which coordinated power reduction in 
wireless sensor networks may lead to increasing its efficiency, mitigating interference, and thus improving the network performance. Using simulations the authors analyze the relationship between transmission power and communication efficiency, in order to determine how appropriate power reduction can improve both energy and spectrum efficiency.

The paper by $\mathrm{L}$. Li et al. also addresses the energy consumption, but analyzing the network efficiency in terms of information dissemination. This work presents a novel and efficient model for studying the effect of nodes on information diffusion over mobile social networks. The proposal is based on the universal generating function method and the discrete stress-strength interference theory. In this model, the effect of the users on information diffusion is quantified as node susceptibility, and, based on that, the proposed model helps various decision-makers identify the most susceptible nodes to the corresponding information at different time periods.

The paper by T. Shin J. Byun presents a social-enabler (SEnabler) middleware architecture for supporting the development of social IoT-based smart systems. This middleware helps convert the existing objects into social objects, and it was used for supporting the activities of vehicle social networks focusing on its energy saving. By using sharing and competition strategies the energy saving service can reduce energy consumption by up to $31.7 \%$ per node.

The mobile collaborative systems can impact several applications domains; particularly, the papers by D. E. Arias et al. and A. Neyem et al. present two systems focused on healthcare. The first paper introduces a mobile sensing device for supporting mobility of people using wheelchair. These chairs are instrumented with various sensors to get information from the user pressure relief habits, activity levels, vital signs, and ambient conditions. The results of a case study performed during two weeks, involving six fulltime wheelchair users, indicate the system is able to capture pressure relief patterns and tilt-in-space usage, a mechanism required to avoid pressure ulcers. With a different goal, the paper of A. Neyem et al. presents a cloud-based mobile system for supporting team collaboration, risk evaluation, and decision-making while patients in critical health condition are transported between health centers. This system helps local and remote medical personnel to stay aware of the patients' status in real time and take preventive actions and early response activities when required. Thus, the system intends to increase the safety of the interhospital transfers.

Collectively, this corpus of knowledge advances the state of the art in the mobile collaborative systems domain, and it provides insights for new researches and applications in this area.

\author{
Sergio F. Ochoa \\ Weiming Shen \\ Giancarlo Fortino \\ Wenfeng Li
}



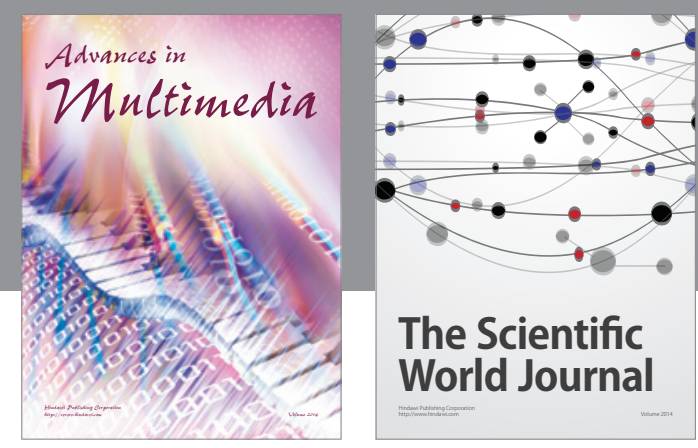

The Scientific World Journal
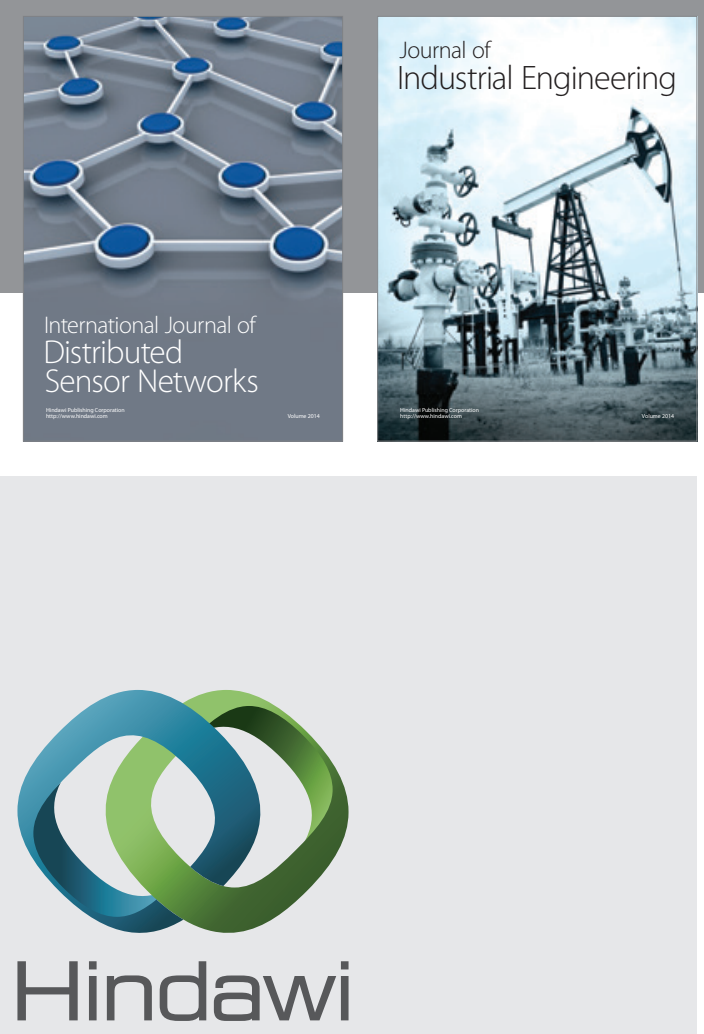

Submit your manuscripts at

http://www.hindawi.com

\section{Computer Networks} and Communications
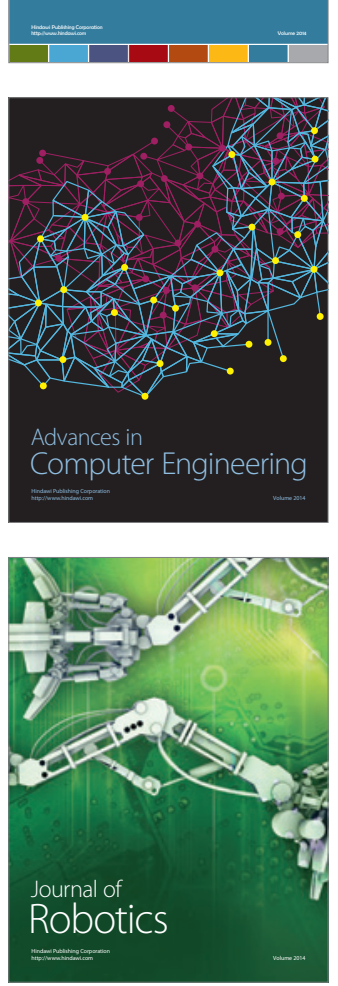
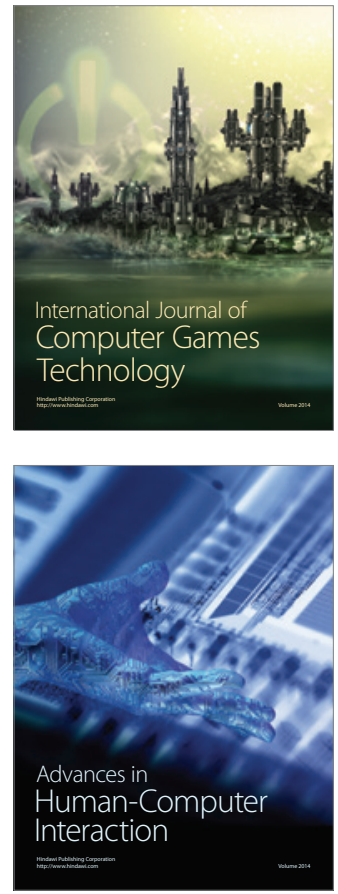
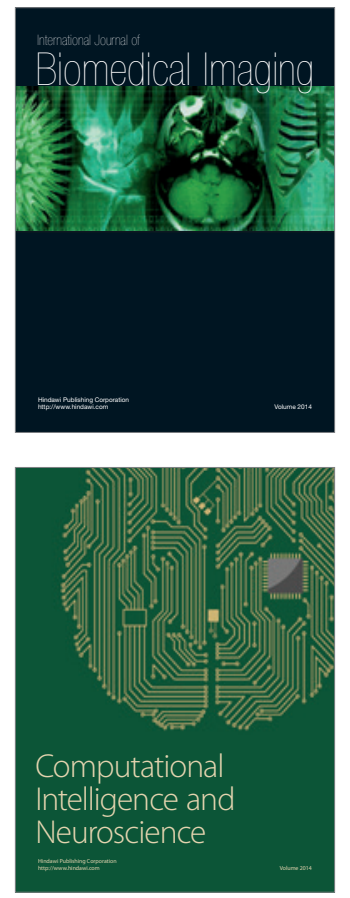
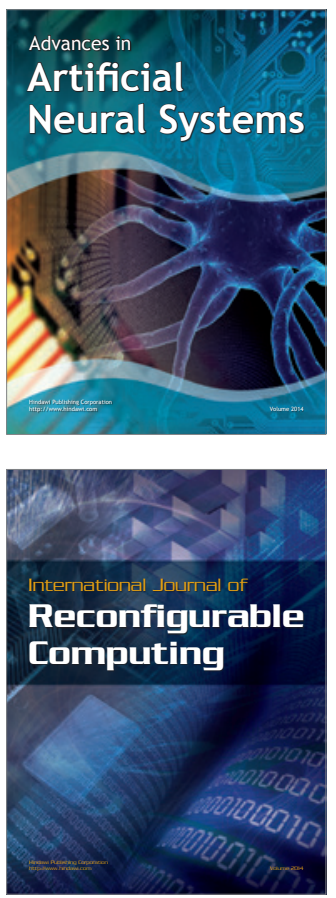
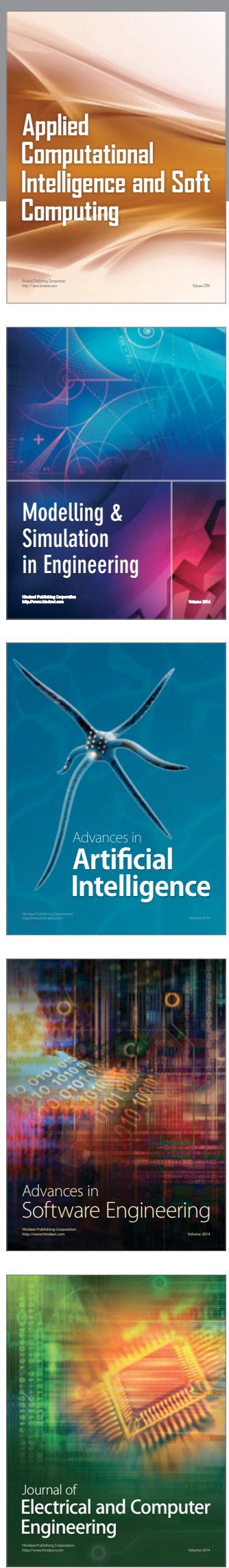